\title{
La importancia de comprender los vínculos entre naturaleza y sociedad
}

\section{Mateo Aguado Caso'}

Resumen: La naturaleza contribuye a la calidad de vida de las personas mediante una gran variedad de formas, constituyendo, al fin y al cabo, la verdadera base del bienestar y de la subsistencia humana. Sin embargo, la globalización capitalista bajo la cual vivimos está favoreciendo el avance de unos estilos de vida cada vez más acelerados y contaminantes que están afectando gravemente a la estructura y funcionamiento de los ecosistemas de nuestro planeta. Este hecho, como han alertado numerosos trabajos científicos recientemente, podría estar empujándonos hacia un punto crítico de no retorno que, de seguir así, podría desembocar durante las próximas décadas en diversos colapsos ecológico-sociales que podrían acabar por comprometer tanto la integridad ecológica de nuestro planeta como el bienestar y la supervivencia de gran parte de la humanidad. Bajo el actual contexto de crisis socio-ecológica en el que vivimos, y en un momento histórico en el que la mayor parte de las decisiones políticas están fuertemente subordinadas al crecimiento de la esfera económica, se hace más necesario que nunca repensar la educación del nuevo milenio para fomentar una nueva concepción de desarrollo humano que sea socialmente justa y ecológicamente sostenible. $Y$ este enorme desafío comienza por comprender que nuestro bienestar y nuestra supervivencia dependen plenamente de los múltiples beneficios que los ecosistemas (cuando están sanos) son capaces de proporcionarnos.

Palabras clave: Desarrollo humano, Educación para la sostenibilidad, Servicios de los ecosistemas, Sostenibilidad socio-ecológica, Bienestar humano.

Fecha de recepción: 14 de enero de 2019.

Fecha de admisión definitiva: 26 de octubre de 2019.

' Laboratorio de Socio-Ecosistemas. Departamento de Ecología. Universidad Autónoma de Madrid. 
Vivir de acuerdo con la naturaleza es el camino más rápido hacia la satisfacción.

DIÓGENES (412-323 a.C.) The importance of understanding the links
between nature and society

Abstract: Nature contributes to human quality of life in many ways, ultimately providing the true basis for human subsistence and wellbeing. However, the capitalist globalization under whose influence we live encourages the development of increasingly accelerated and polluting lifestyles that seriously affect the structure and functioning of our planet's ecosystems. Unless that changes, it could push us towards a critical point of no return, as many scientific studies have lately been indicating, which might lead to scenarios of ecological and social collapse in the coming decades that could ultimately compromise both the ecological integrity of our planet and the well-being and survival of a large part of humanity. In the current context of the socio-ecological crisis in which we live, and in a historical moment in which most political considerations are subservient to economic growth, it is more necessary than ever to rethink education in the new millennium in order to promote a new concept of human development that is socially just and ecologically sustainable. This enormous challenge begins with an understanding that our well-being and survival depend entirely on the multiple benefits that ecosystems (when they are healthy) are able to bestow on us.

Key words: Human development, sustainability education, ecosystem services, socio-ecological sustainability, human well-being.

\section{L'importance de comprendre les liens entre nature et société}

Résumé: La nature contribue à la qualité de vie de l'homme de multiples façons, constituant en fin de compte la véritable base du bien-être et de la subsistance de l'homme. Cependant, la mondialisation capitaliste à laquelle nous sommes témoin favorise le développement de modes de vie de plus en plus accélérés et polluants qui affectent sérieusement la structure et le fonctionnement des écosystèmes de notre planète. Ce fait, comme l'ont récemment mis en garde de nombreux travaux scientifiques, pourrait nous pousser vers un point critique de non-retour qui, s'il se poursuit ainsi, pourrait conduire au cours des prochaines décennies à divers effondrements écologiques et sociaux qui pourraient finir par compromettre à la fois l'intégrité écologique de notre planète et le bien-être et la survie d'une grande partie de I'humanité. Dans le contexte actuel de crise socio-écologique dans lequel nous vivons, et dans un moment historique où la plupart des décisions politiques sont fortement subordonnées à la croissance économique, il devient plus que jamais nécessaire de repenser leéducation du nouveau millénaire afin de promouvoir une nouvelle notion du développement humain qui soit socialement juste et écologiquement durable. Et cet énorme défi commence par la compréhension du fait que notre bien-être et notre survie dépendent entièrement des multiples avantages que les écosystèmes (lorsqu'ils sont en bonne santé) sont capables d'offrir.

Mots clé: Développement humain, éducation à la durabilité, services éco-systémiques, durabilité socio-écologique, bien-être humain. 


\section{Introducción}

El reconocido biólogo estadounidense Edward $\mathrm{O}$. Wilson viene sosteniendo desde hace tiempo que los seres humanos poseemos la necesidad innata de afiliarnos al resto de seres vivos, estando de este modo cognitivamente unidos a la naturaleza (Wilson, 1984). Esta idea, conocida como la "hipótesis de la biofilia", sugeriría así que nuestra reciente separación del mundo natural nos ha llevado a vivir en ambientes mayoritariamente urbanos sin que hayamos borrado aún nuestro aprendizaje sobre el valor que la naturaleza tiene para nuestras vidas. Con ello, la generalización de unos estilos de vida cada vez más sedentarios, urbanitas y tecno-dependientes nos estaría haciendo olvidar en los albores del Antropoceno (Aguado, 2017) que nuestra calidad de vida y nuestro bienestar están estrechamente ligados a la salud de los ecosistemas (Aguado, 2018; EME, 2011).

Bajo este enfoque, las alteraciones que las actividades humanas están provocando sobre el funcionamiento global de la biosfera (como el cambio climático, la sobreexplotación de recursos geóticos y bióticos, la contaminación o los cambios de uso de suelo) (Duarte et al., 2009; Stefen et al., 2004) han de tener, más tarde o más temprano, un efecto directo y reconocible sobre el bienestar humano (Díaz et al., 2006; MA, 2005a). Aunque en algunos casos este efecto pueda ser positivo a corto plazo y para algunos grupos sociales en concreto (por ejemplo, la sobreexplotación de un ecosistema forestal puede aumentar de forma temporal el bienestar económico y material de algunas personas), los enormes cambios que los seres humanos estamos imprimiendo sobre los sistemas naturales de nuestro planeta (Rockström et al., 2009) se traducen, la inmensa mayoría de las veces, en un descenso importante en el flujo de servicios que los ecosistemas pueden proporcionarnos (MA, 2005a), contribuyendo así -en el medio y largo plazonegativamente a nuestra calidad de vida.

\section{Los servicios de los ecosistemas}

Los ecosistemas y la biodiversidad contribuyen al bienestar de las personas mediante una gran variedad de formas. Un concepto útil para el estudio de todas estas contribuciones es el de servicios de los ecosistemas, entendido como las contribuciones directas e indirectas que los ecosistemas hacen a las diferentes componentes del bienestar humano (De Groot et al., 2014; EME, 2011) y que hacen que la vida sea posible y merezca la pena (Díaz et al., 2006).

Aunque obviamente los servicios de los ecosistemas no son de por sí suficientes para proporcionar bienestar humano, (Butler y Oluoch-Kosura, 2006), se entienden 
como una parte imprescindible del mismo, especialmente cuando analizamos el bienestar a largo plazo (Daily, 1997).

Los servicios de los ecosistemas se clasifican en tres grandes categorías (EME, 2011; MA, 2005a): i) los servicios de abastecimiento (que son los productos obtenidos directamente de los ecosistemas, como el alimento, el agua, las medicinas naturales - la madera); ii) los servicios de regulación (que se obtienen de manera indirecta a partir de los procesos de los ecosistemas, como la calidad del aire y del agua - la regulación climática); y iii) los servicios culturales (que son los beneficios no materiales que la gente obtiene estando en contacto directo con los ecosistemas a través del enriquecimiento espiritual, el disfrute estético, el desarrollo cognitivo o las actividades recreativas). Tal y como se muestra en la Figura 1, todos estos servicios contribuyen a la calidad de vida de las personas proporcionando diferentes beneficios sobre las principales dimensiones que tiene el bienestar humano: los materiales básicos para una vida buena, la salud, las buenas relaciones sociales, la seguridad, y la libertad de acción y elección (EME, 2011; MA, 2005a).

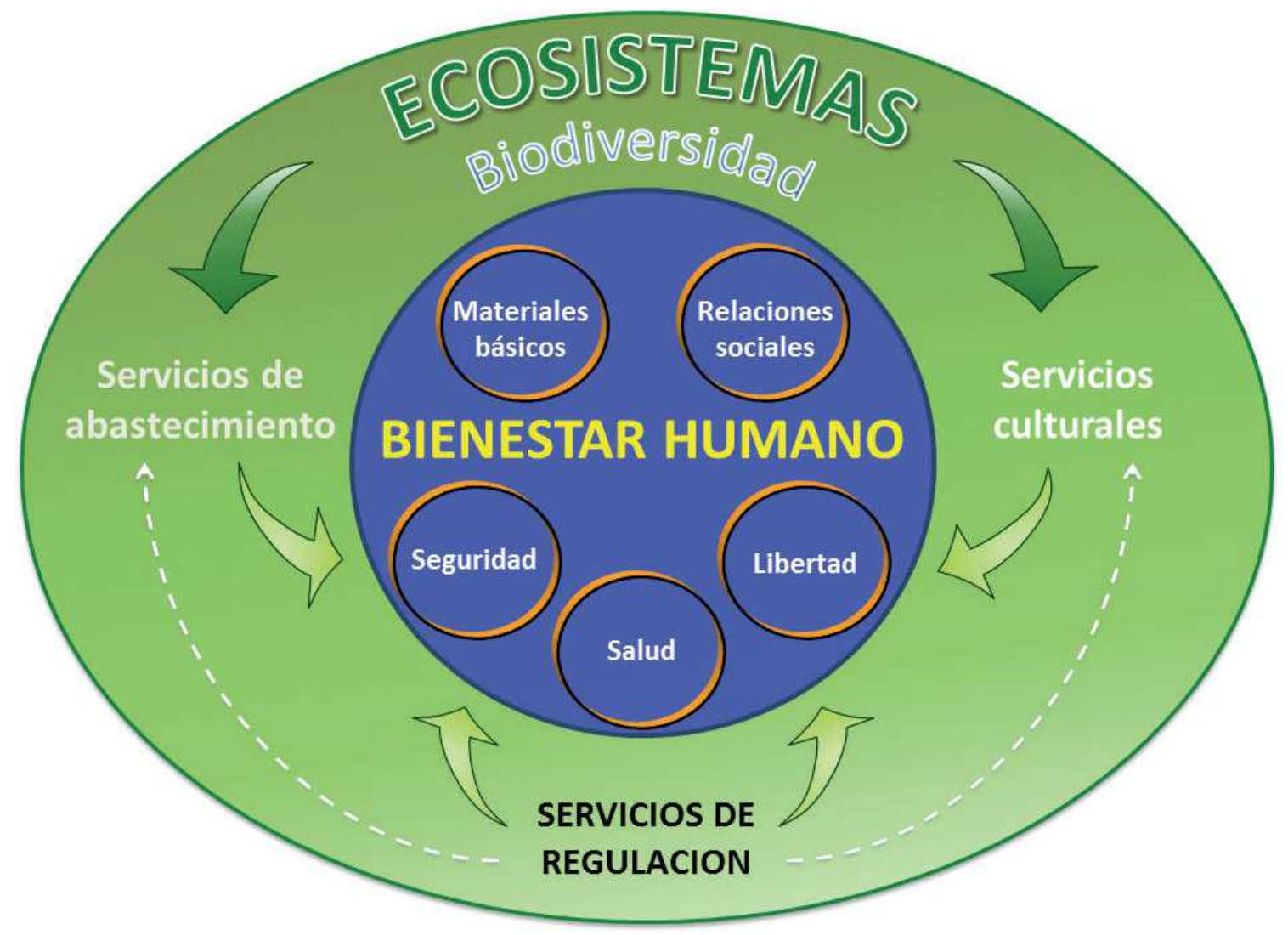

FIGURA 1. Las diferentes componentes del bienestar humano dependen, en mayor o menor medida, y de forma directa e indirecta, de las tres grandes categorías de servicios que proporcionan los ecosistemas: los servicios de abastecimiento, los servicios culturales $y$, fundamentalmente, los servicios de regulación. Modificado de EME (2011). 
Los servicios de los ecosistemas se presentan de esta forma como un puente de unión entre los sistemas naturales y los sistemas sociales a través del cual se reconoce que nuestro bienestar y el de las generaciones futuras depende -en gran medida- de la integridad ecológica y de la conservación de la biodiversidad. De este modo se conceptualizan los ecosistemas como la verdadera base de la subsistencia humana $y$, en definitiva, como la base del bienestar humano (EME, 2011).

Este concepto ha favorecido además un importante cambio de paradigma en lo que respecta a las motivaciones conservacionistas, que en los últimos años han pasado de los argumentos emotivos y éticos (asentados en los valores intrínsecos de la naturaleza) (McCauley, 2006) a los argumentos más pragmáticos y utilitarios, que tienen en cuenta las contribuciones de los ecosistemas a la calidad de vida de las personas (Costanza et al., 1998; Daily et al., 1997). Esta nueva perspectiva facilita la comprensión de los estrechos vínculos que existen entre la naturaleza y la sociedad, ayudando con ello a entender mejor cómo los profundos cambios que están sucediendo actualmente en la biosfera fruto de las actividades humanas han de tener-más tarde o más temprano- repercusiones negativas sobre el bienestar de las personas (al ser alterados los servicios que los ecosistemas proporcionan) (Martín-López y Montes, 2014).

\section{Los vínculos entre los servicios de los ecosistemas y el bien- estar humano}

La Evaluación de los Ecosistemas del Milenio (MA, 2005a) es probablemente la obra más completa en cuanto a la articulación del bienestar humano en un contexto socio-ecológico (King et al., 2014). Este proyecto, coordinado por Naciones Unidas y elaborado en base al trabajo de más de mil expertos de casi un centenar de países diferentes, ha supuesto la auditoría ecológica con base científica más importante que se ha llevado a cabo hasta la fecha sobre los ecosistemas del planeta y sus relaciones con el bienestar humano (EME, 2011).

Desde el importante impulso que supuso este proyecto, han sido muchos los científicos que han investigado las conexiones existentes entre los servicios de los ecosistemas y el bienestar humano (Aguado et al., 2018; Butler y Olvoch-Kosura, 2006; Haines-Young y Potschin, 2010; King et al., 2014; Sandifer et al., 2015; Summers et al., 2012). Estas conexiones se caracterizan, entre otras cosas, por ser enormemente complejas y diversas (Salzman et al., 2001), así como por suceder a diferentes escalas tanto espaciales como temporales (MA, 2003). Por ello, y a pesar de los notables avances obtenidos durante los últimos años, aún hay un 
largo camino por recorrer en esta materia, pues todavía no comprendemos bien del todo cómo los diferentes servicios de los ecosistemas contribuyen a mejorar las diferentes componentes del bienestar humano (Delgado y Marín, 2016; Jax y Heink, 2015). En cualquier caso, y tal y como reconocen Mooney et al. (2004), la Evaluación de los Ecosistemas del Milenio ha generado un marco conceptual clave para guiar la comprensión y la medición del bienestar en el contexto amplio de los sistemas socio-ecológicos.

Formalizar el concepto de bienestar y sus diferentes dimensiones se convierte así en algo esencial para poder comprender mejor las aportaciones que los ecosistemas hacen a los diferentes aspectos de la vida humana (Polishchuk y Rauschmayer, 2012; Summers et al., 2012). Fomentar durante las próximas décadas el debate, la investigación y la educación sobre los diferentes componentes del bienestar humano nos permitirá ampliar la comprensión de los múltiples factores ambientales que lo alimentan, abriéndonos con ello la posibilidad de explorar mejoras en la calidad de vida de las personas que no impacten negativamente sobre los ecosistemas y sus servicios (Roberts et al., 2015).

Aunque durante los últimos años han surgido varias propuestas alternativas al respecto (ver, por ejemplo, Vemuri y Costanza, 2006, Smith et al., 2013y Summers et al., 2014), los aspectos fundamentales del bienestar que siguen utilizándose en la actualidad como referencia en las investigaciones socio-ecológicas son las cinco dimensiones que propuso a comienzos del presente siglo la Evaluación de los Ecosistemas del Milenio; a saber: i) materiales básicos para una vida buena, ii) salud, iii) buenas relaciones sociales, iv) seguridad, yv) libertad de acción y elección (MA, 2005a). Esta ordenación será tomada como referencia a continuación para analizar -más detalladamente- las diversas contribuciones que los ecosistemas realizan a las diferentes dimensiones del bienestar humano.

\section{I. Materiales básicos para una vida buena}

La relación entre los servicios de los ecosistemas y los materiales básicos ha sido uno de los vínculos socio-ecológicos más investigados históricamente. Esta dimensión del bienestar está fundamentalmente ligada a los servicios de abastecimiento (principalmente alimentos y agua dulce) y a algunos servicios de regulación, como la purificación hídrica, la polinización, el control de plagas o la fertilidad de los suelos (MA, 2003). Los ecosistemas también proporcionan materiales como la madera, la turba o los combustibles fósiles que son de gran importancia para la construcción de los hogares y para la obtención de fuentes de calor dentro de ellos. 
Dado que los materiales básicos para una vida buena son la dimensión del bienestar más estrechamente ligada a la capacidad de subsistencia, resulta lógico que un mal funcionamiento de los ecosistemas pueda relacionarse con situaciones indeseadas de hambrunas e inanición al verse degradados servicios básicos como el suministro de alimentos o de agua potable (MA, 2005b). Actualmente, la creciente escasez de agua dulce y la desigual producción de alimentos están teniendo impactos negativos sobre amplios sectores humanos que amenaza con poner en riesgo la salud y la supervivencia de miles de personas en todo el mundo (MA, 2005a).

En esta línea, algunos estudios han defendido que el colapso de algunas civilizaciones antiguas (como Mesopotamia o el imperio Maya, entre otras) se pudieron deber en gran medida a la sobreexplotación de los servicios de los ecosistemas (fundamentalmente los de abastecimiento) (Diamond, 2005; Tainter, 1990). Trabajos como el de Hamilton y Clemens (1999) han llegado a sugerir que los descensos de bienestar vividos en las últimas décadas por algunas regiones del mundo (como el África subsahariana) podrían explicarse, al menos en parte, por el deterioro experimentado en los servicios de estos ecosistemas.

\subsection{Salud}

Las conexiones entre la salud humana (tanto física como psicológica) y los ecosistemas han sido ampliamente documentadas durante las últimas décadas por múltiples investigaciones científicas (ver, por ejemplo, Conn, 1998; Corvalan et al., 2005; Maller et al., 2002 y McMichael et al., 2005).

Son fundamentalmente los servicios de abastecimiento y los servicios de regulación los que más estrechamente se vinculan a la salud física de las personas (MA, 2005a). Así, el deterioro de importantes servicios de regulación como la calidad del agua y la calidad del aire está detrás de la propagación de muchas enfermedades infecciosas (MA, 2003, 2005b). Igualmente, servicios de abastecimiento esenciales para la vida como la producción de alimentos o el suministro de agua dulce tienen una importancia capital en la salud física, pues cuando su suministro decae existe el peligro de enfermar y de caer en situaciones de hambruna y desnutrición (MA, 2003). Tal y como destaca el MA (2005a), alrededor de una cuarta parte de la carga de enfermedades en los países más pobres del mundo es achacable actualmente a la desnutrición. Igualmente, las enfermedades derivadas del consumo de agua inadecuada se estima que originan 1,7 millones de muertes al año, provocando la pérdida de al menos 54 millones de años de vida sana al año (MA, 2005a). 
Otro servicio de abastecimiento directamente relacionado con la salud física (sobre todo en los ambientes rurales e indígenas) es el que tiene que ver con las medicinas naturales obtenidas a partir de plantas silvestres. A día de hoy el $80 \%$ de la población mundial utiliza medicinas naturales (Roberts et al., 2015) y aproximadamente el $50 \%$ de todos los medicamentos recetados en el mundo tienen su origen en plantas (MA, 2005a). Según cálculos de Robertson (2008), 118 de los 150 medicamentos más utilizados en EEUU se obtienen actualmente a partir de fuentes naturales.

Aunque con menor fuerza y notoriedad, la salud también presenta vínculos con los servicios culturales, a través, fundamentalmente, de los beneficios recreativos y espirituales (MA, 2003, 2005a). Numerosos estudios han puesto de manifiesto como observar e interactuar con la naturaleza de forma frecuente (bien sea a través de su mera contemplación o mediante actividades de ocio y recreo) tiene efectos beneficiosos (tanto emocionales como fisiológicos) sobre la salud de las personas, asociándose al disfrute de emociones positivas y a un mayor bienestar (Carlisle et al., 2009; Feral, 1998; Ferrer-i-Carbonell y Gowdy, 2007; Kaplan, 1992; Kjell, 2011 ; Korpela y Kinnunen, 2010; Leather et al., 1998; Lewis, 1996; Nisbet et al., 2008, 2011; Van Den Berg et al., 2007). Por ejemplo, vivir cerca de espacios verdes se ha asociado con una mejor salud (De Vries et al., 2003), con un mejor bienestar mental (Maas et al., 2009; Van den Berg et al., 2010; White et al., 2013) y con un menor riesgo de padecer enfermedades (Maas et al., 2009), mientras que, por su parte, altos niveles de contaminación del aire se han asociado con una menor satisfacción con la vida (Silva y Brown, 2013; Welsch, 2006).

Bell et al. (2008) detectaron que los niños y jóvenes que vivían en barrios con más áreas verdes presentaban menor índice de masa corporal, presumiblemente debido al aumento de la actividad física al aire libre. En un sentido similar Fjortoft (2004) descubrió que los niños que disponían de paisajes naturales para jugar presentaban mejores aptitudes motrices, de equilibrio y de coordinación que aquellos que crecían en ambientes más urbanizados. Un estudio realizado en Japón mostró que el acceso a los espacios verdes en las áreas urbanas tenía beneficios significativos en la longevidad de las personas mayores (Takano et al., 2002).

Pero las relaciones entre la salud y los servicios culturales no se limitan sólo al ámbito físico (como el ejercicio o las actividades recreativas en entornos naturales) sino que también atañen al ámbito psicológico. Tanto es así que desde mediados de la década de los 90 existe toda una disciplina científica dedicada al estudio de cómo la salud psicológica se entrelaza con el estado de conservación de la naturaleza: la ecopsicología (Conn, 1998). Esta disciplina ha puesto de manifiesto, entre otros hallazgos, como las personas obtenemos beneficios psicológicos al interaccionar con otras especies (Ferrer-i-Carbonell y Gowdy, 2007; Kellert y 
Wilson, 1995). De este modo, y tal y como sugieren Winter y Koger (2004), se podría entender que la acelerada pérdida de biodiversidad que estamos viviendo actualmente (Barnosky et al., 2011) podría tener consecuencias negativas para la psique humana.

Un famoso trabajo de la década de los 80 reveló que las tasas de recuperación de los pacientes sometidos a cirugía fueron mucho mayores entre aquellas personas que disponían de vistas a la naturaleza desde sus ventanas (Ulrich, 1984). Por su parte, Kaplan y Kaplan (1989) mostraron que el acceso a la naturaleza en el lugar de trabajo se relaciona positivamente con niveles más bajos de estrés laboral y con mayores niveles de satisfacción. Además, los empleados que cuentan con vistas a la naturaleza desde su oficina suelen reportar menos enfermedades y menos dolores de cabeza. En general, tal y como recogen Rohde y Kendle (1994), la contemplación de paisajes de naturaleza -y en particular los provistos de aguaha demostrado tener una influencia beneficiosa sobre el estado psicológico de las personas, contribuyendo al incremento de los sentimientos de placer, a la recuperación de la fatiga mental y a la disminución de emociones negativas como la ira, la ansiedad o el estrés. De forma similar, Wells y Evans (2003) informaron sobre el efecto amortiguador del estrés que la proximidad a la naturaleza tenía sobre los niños crecidos en ambientes rurales.

Según alertan algunos autores, nuestra creciente desconexión con la naturaleza (ilustrada a través de unos estilos de vida cada vez más urbanos y tecno-dependientes) estaría favoreciendo entre los más jóvenes la propagación del conocido como "trastorno por déficit de naturaleza", un mal relacionado con la obesidad, la depresión y el déficit de atención (Kellert, 2005; Louv, 2008; Taylor y Kuo, 2009). Ante un mundo cada vez más urbanizado y desconectado de los ecosistemas, y en donde las tasas de enfermedades relacionadas con el estrés y la depresión no dejan de aumentar año tras año (Maller et al., 2002), se vislumbra fundamental para el bienestar y la salud humana recuperar nuestra conexión cultural y cotidiana con la naturaleza.

\subsection{Buenas relaciones sociales}

Los vínculos entre los seres humanos y la naturaleza son diferentes en cada lugar y están sujetos, como es lógico, a diversas particularidades ecológicas. Estas particularidades pueden llegar a condicionar el tipo de relación social predominante entre los diferentes grupos humanos (MA, 2003). Así, por ejemplo, las relaciones sociales que presentan las asociaciones de pescadores difieren en muchos aspectos de las que exhiben los colectivos de pastores nómadas o las sociedades agrícolas (MA, 2003). Los ecosistemas, por tanto, pueden influir en 
el tipo de relaciones sociales que los diferentes grupos humanos construyen sobre ellos con el paso del tiempo.

Los ejemplos más claros sobre la vinculación existente entre los ecosistemas y las buenas relaciones sociales suelen aportarlo las comunidades rurales e indígenas, pues son estos los grupos sociales que viven más estrechamente unidos a la vida silvestre (siendo, además, los grupos más vulnerables ante cualquier tipo de deterioro en los ecosistemas) (MA, 2005a). Tal y como señala el propio MA (2005a), los cambios en los servicios de abastecimiento y regulación pueden afectar a las relaciones sociales, principalmente a través de sus efectos más directos sobre el bienestar material, la salud y la seguridad.

Uno de los ejemplos más paradigmáticos para ilustrar las conexiones entre la biodiversidad y las buenas relaciones sociales lo aporta el caso de los buitres Gyps y las comunidades parsi en la India. Según recogen Markandya et al. (2008), el enorme descenso experimentado en los últimos años en las poblaciones de estas aves carroñeras en la India (un descenso que se estima podría llegar a ser del 95\% para algunas especies de buitre) (Pain et al., 2003; Prakash et al., 2003) llegó a originar profundas divisiones entre los habitantes de la religión parsi sobre cómo deshacerse de sus muertos, pues el descenso en las poblaciones de buitres impedía colocar los cadáveres -como dicta la creencia parsi- en las conocidas Torres del silencio ly es que la acumulación y descomposición de los cuerpos a la intemperie, sin buitres que se alimenten de ellos, suponía un importante foco de problemas para la salud pública) (Markandya et al., 2008).

Los servicios de los ecosistemas más estrechamente vinculados a las relaciones sociales son los culturales (MA, 2003), sobre todo los relacionados con el disfrute espiritual y estético de los paisajes naturales y los relacionados con las actividades recreativas y de ocio. Los espacios naturales promueven el aumento de las oportunidades para la interacción social (Coley et al., 1997; Roberts et al., 2015). De este modo, tal y como argumenta Kuo (2011), las relaciones sociales tienden a mejorar en entornos verdes y abiertos al promover comportamientos pro-sociales y ayudar a mitigar algunas de las conductas negativas asociadas a la vida urbana. Además, la acción colectiva necesaria para proteger los ecosistemas locales puede ser en sí misma una importante fuerza de unión y cohesión social, ayudando a asentar sentimientos de identidad hacia la comunidad (cuyos habitantes comparten el objetivo común de hacer de su entorno un mejor lugar para vivir) (EPA, 1997). La educación ambiental y el conocimiento ecológico local, por su parte, favorecen la construcción de vínculos sociales y la generación de cohesión social ligada a los ecosistemas. 


\subsection{Seguridad}

Los vínculos entre la seguridad y los servicios de los ecosistemas, aunque aún no han sido muy estudiados, tienen una tremenda trascendencia sobre el bienestar humano. La seguridad suele verse afectada tanto por los cambios en los servicios de abastecimiento (que alteran el suministro de materiales esenciales como los alimentos y el agua) como por los cambios en los servicios de regulación (que influyen en la frecuencia y en la magnitud de desastres como las inundaciones, las sequías o los deslizamientos de tierra) (MA, 2003, 2005a). Además, los cambios en los servicios de abastecimiento están detrás de numerosos conflictos locales, nacionales e internacionales en torno al control de recursos escasos y estratégicos (MA, 2005a; Le Billon, 2001). Un ejemplo claro de cómo los servicios de abastecimiento pueden alterar el orden social y causar inseguridad lo encontramos en la conocida guerra del agua de Cochabamba, un conflicto que tuvo lugar en el año 2000 en Bolivia y cuyo detonante fue la privatización del abastecimiento de agua potable por parte del gobierno municipal.

Por su parte, los vínculos entre los servicios de regulación y la seguridad son igualmente notorios. Por ejemplo, los bosques nativos y los pastizales de montaña interceptan la lluvia y aumentan su infiltración en las aguas subterráneas, lo que reduce el riesgo de deslizamientos de tierras y contribuye a reducir la erosión (lo cual, a su vez, reduce la acumulación de sedimentos en los cauces de los ríos, reduciendo así el riesgo de inundaciones) (Roberts et al., 2015).

Importantes servicios de regulación como el control de plagas, la regulación frente a amenazas naturales, el control de la erosión o la regulación climática (mediante el secuestro de carbono por parte de la vegetación) tienen importantes vínculos con la seguridad humana (MA, 2005a). Así, el desajuste del clima que actualmente está experimentando nuestro planeta fruto de las emisiones humanas de $\mathrm{CO}_{2}$ representa una seria amenaza para millones de personas en todo el mundo (según la ONU para el año 2050 el número de desplazados climáticos podría alcanzar la cifra de 200 millones de personas). Los eventos extremos asociados al cambio climático (como los ciclones, las inundaciones, las sequías o las olas de calor), a pesar de no haber sido hasta ahora bien reportados, constituyen un caso claro de cómo el deterioro en el funcionamiento de los ecosistemas puede comprometer de forma directa nuestra integridad física y, por ende, nuestro bienestar. Por citar sólo algunos ejemplos, las fuertes inundaciones que en 2013 causaron los monzones en la India provocaron la pérdida de más de 5.000 vidas humanas, y la ola de calor que azotó Europa en 2003 se calcula que, al menos, provocó en torno a 30.000 muertes prematuras. Frente a un escenario de incremento en la fusión de hielos y glaciares y de aumento del nivel del mar (IPCC, 2007), la creciente ocupación 
humana de llanuras aluviales y zonas costeras está igualmente incrementando nuestra vulnerabilidad ante el cambio climático.

Los servicios culturales, por su parte, también han mostrado tener contribuciones importantes sobre la seguridad. Así, Kuo y Sullivan (2001) relacionaron la existencia de espacios verdes en las zonas urbanas con una reducción en la criminalidad y la delincuencia. Por su parte, Cackowski y Nasar (2003) mostraron cómo la ornamentación vegetal utilizada en los márgenes y medianas de las carreteras influía positivamente en la seguridad vial, promoviendo en los conductores la reducción de la velocidad, transmitiendo un efecto calmante y disminuyendo su fatiga.

\subsection{Libertad de acción y elección}

Los vínculos entre la libertad y los ecosistemas, aunque algo más difíciles de identificar, son igualmente fundamentales, pues se relacionan transversalmente con el resto de dimensiones del bienestar humano. El ejemplo más claro de esto lo encontramos en la libertad respecto al uso del tiempo. Así, tal y como recoge el MA $(2005 a, b)$, el descenso en la provisión de leña y agua potable como resultado del deterioro de los ecosistemas ha demostrado tener repercusiones negativas sobre la libertad de acción y elección en comunidades rurales, pues aumenta la cantidad de tiempo necesario para cubrir -mediante la recolección- dichas necesidades básicas, disminuyendo con ello el tiempo disponible para importantes aspectos del bienestar como la educación o la atención a otros miembros de la familia.

Tal y como han detectado varios autores, disponer de tiempo libre contribuye positivamente a la salud física y mental de las personas (Krueger et al, 2009; Williams y Patterson, 2008) pues, entre otras cosas, propicia un distanciamiento psicológico del trabajo (Sonnentag y Binnewies, 2010) y posibilita las interacciones sociales y la mejora de la cohesión social (Smith et al., 2013). Además, según Korpela y Kinnunen (2010), invertir este tiempo libre en interactuar con una naturaleza sana se correlaciona significativamente con la satisfacción de vida y con la relajación, aspectos ambos que contribuyen positivamente al bienestar subjetivo y a la salud.

El buen funcionamiento de algunos servicios de abastecimiento y de regulación tiene una relación directa con el cumplimiento de los derechos humanos más básicos. Así, el derecho a una alimentación adecuada (Art. 25 de la Declaración Universal de los Derechos Humanos) está intrínsecamente ligado a los servicios de abastecimiento (como proveer alimentos y agua dulce) así como a servicios de regulación (como mantener la regulación hídrica y la fertilidad de los suelos). 
Los servicios culturales, por su parte, presentan vínculos con la libertad de acción y elección a través, fundamentalmente, de la educación. Servicios como el conocimiento ecológico local, el conocimiento científico o la educación ambiental, brindan grandes oportunidades de aprendizaje para la expansión de las libertades a través del conocimiento (Smith et al., 2013). La investigación y la transferencia formal e informal de estos conocimientos conduce a una mayor comprensión de cómo funcionan los ecosistemas y cómo nuestras acciones cotidianas afectan a la provisión de estos servicios esenciales para nuestro bienestar (Roberts et al., 2015).

Según han evidenciado varios autores, la interacción con los ecosistemas en edades tempranas se relaciona con mejoras en las capacidades cognitivas y en la resolución de problemas (Kahn y Keller, 2002; Wells, 2000), aspectos que repercuten positivamente sobre la educación. El conocimiento ecológico local, por su parte, es fundamental en la aportación de los relatos históricos que alimentan las tradiciones y la identidad rural. Estos relatos, además, contribuyen muchas veces a la investigación científica y a la correcta gestión del medio natural local, así como a que el conocimiento no escrito se transmita de generación en generación (Huntington, 2000).

\section{Conclusiones: hacia un bienestar sostenible}

Como hemos visto, los beneficios que los seres humanos obtenemos de la naturaleza son muchos y muy variados. Todos estos beneficios ponen de manifiesto una realidad difícilmente cuestionable: que del buen estado y funcionamiento de los ecosistemas depende nuestra subsistencia y nuestro bienestar presente y futuro, pues la biodiversidad y los servicios de los ecosistemas constituyen la base biofísica sobre la cual se asienta el resto de capitales de origen humano (EME, 2011 ). La sostenibilidad socio-ecológica y el bienestar humano son, por lo tanto, dos nociones hermanadas que emanan de una misma interacción dinámica. Son, al fin y al cabo, dos caras de una misma moneda (Aguado y Riechmann, 2013).

Sin embargo, la globalización urbana bajo la cual vivimos en los albores del siglo XXI está favoreciendo el avance de unos estilos de vida acelerados, contaminantes y despilfarradores que encuentran en las ciudades su verdadera razón de ser (Gleeson, 2012; Montes y Duque, 2013). Este fenómeno está impulsando en el mundo entero la propagación de una serie de patrones de consumo y pautas de comportamiento que están alejando a los seres humanos de sus tradicionales ritmos de vida al tiempo que nos desconectan de la naturaleza y nos restan calidad de vida. En una biosfera finita y sujeta a límites planetarios, tratar de ensalzar y defender una noción de vida basada en las aspiraciones materiales y en el cre- 
cimiento continuo puede resultar una mala estrategia en el medio y largo plazo para el bienestar humano.

Bajo el contexto de crisis socio-ecológica en el que nos encontramos se hace más urgente que nunca repensar la educación y la política del nuevo milenio para fomentar una nueva concepción del desarrollo humano que, consciente de la enorme importancia que tiene cuidar los vínculos naturaleza-sociedad, nos permita transitar -a través de la educación- hacia un futuro más justo y sostenible. Necesitamos, a fin de cuentas, aprender a armonizar en el actual contexto de transformaciones antropogénicas el bienestar de todos los seres humanos con el buen estado y funcionamiento de los ecosistemas. Es decir, necesitamos aprender a vivir bien dentro de los límites planetarios.

\section{Referencias}

Aguado, M. (2017) "Llamando a las puertas del antropoceno". Iberoamérica Social: revistared de estudios sociales VII, pp. 42 - 60. Recuperado en http:// iberoamericasocial.com/llamando-a-las-puertas-delantropoceno/.

- (2018) "En las fronteras de la sostenibilidad". Iberoamérica Social: revista-red de estudios sociales IX, pp. 34 - 37. Recuperado en https://iberoamericasocial. com/en-las-fronteras-de-la-sostenibilidad.

Aguado, M., González, J. A., Bellot, K., López-Santiago, C. y Montes, C. (2018) "Exploring subjective well-being and ecosystem services perception along a rural-urban gradient in the high Andes of Ecuador". Ecosystem Services, 34, 1-10.

Barnosky, A. D., Matzke, N., Tomiya, S., Wogan, G. O., Swartz, B., Quental, T. B., ... Y MERSEY, B. (2011) "Has the Earth's sixth mass extinction already arrived?". Nature, 471(7336), 51.

BELL, J. F., WILSON, J. S. y LIU, G. C. (2008) "Neighborhood greenness and 2-year changes in body mass index of children and youth". American journal of preventive medicine, 35(6), 547-553.

ButLer, C. D., y Olvoch-KosuRA, W. (2006) "Linking future ecosystem services and future human well-being". Ecology and Society, 11 (1). 
CACKOWSKI, J. M. y NASAR, J. L. (2003) "The restorative effects of roadside vegetation implications for automobile driver anger and frustration". Environment and Behavior, 35(6), 736-751.

Carlisle, S., Henderson, G. y Hanlon, P. W. (2009) "Wellbeing': A collateral casualty of modernity?". Social Science \& Medicine, 69(10), 1556-1560.

Coley, R. L., Sullivan, W. C. y Kuo, F. E. (1997) "Where does community grow? The social context created by nature in urban public housing". Environment and behavior, 29(4), 468-494.

ConN, S. A. (1998) "Living in the earth: Ecopsychology, health and psychotherapy". The Humanistic Psychologist, 26(1-3), 179-198.

Corvalan, C., Hales, S. y McMichael, A. J. (2005) Ecosystems and human well-being: health synthesis. World Health Organization.

Costanza, R. , d'Arge, R., De Groot, R., Farber, S., Grasso, M., Hannon, B., Limburg, K., Naeem, S., O’Neill, R. V., Paruelo, J., Raskin, R. G., Sutton, P. y Van Den Belt, M. (1998) "The value of the world's ecosystem services and natural capital". Ecological economics, 1(25), 3-15.

DAlly, G. (1997) Nature's services: societal dependence on natural ecosystems. Island Press.

De Groot, D. , Jax, K. y Harrison, P. (2014) "Link between Biodiversity and Ecosystem Services", en M. PotSCHIN y K. JAX (Eds.), OpenNESS Ecosystem Services Reference Book. EC FP7 Grant Agreement n 308428.

De Vries, S., Verheis, R. A., Groenewegen, P. P. y Spreeuwenberg, P. (2003) "Natural environments-healthy environments? An exploratory analysis of the relationship between greenspace and health". Environment and Planning A, 35, 1717-1731.

Delgado, L. E., y Marín, V. H. (2016) "Well-being and the use of ecosystem services by rural households of the Río Cruces watershed, southern Chile". Ecosystem services, $21,81-91$.

DIAMOND, J. (2005) Collapse: How societies choose to fail or succeed. Penguin.

Díaz, S., Fargione, J., Chapin, F. S. y Tilman, D. (2006) "Biodiversity loss threatens human well-being". PLoS biology, 4(8), 1300-1305. 
Duarte, C. M., Alonso, S., Benito, G., Dachs, J., Montes, C., Pardo, M., Rios, A. F., Simó, R. y Valladares, F. (2009) Cambio Global: Impacto de la Actividad Humana sobre el Sistema Tierra. Colección Divulgación, CSIC.

EME (Evaluación de los Ecosistemas del Milenio de España) (2011) La Evaluación de los Ecosistemas del Milenio de España. Síntesis de resultados. Fundación Biodiversidad. Ministerio de Medio Ambiente y Medio Rural y Marino.

epa (United States Environmental Protection Agency) (1997) Community-Based Environmental Protection: A Resource Book for Protecting Ecosystems and Communities. Washington, DC.

FERAL, C. H. (1998) "The connectedness model and optimal development: Is ecopsychology the answer to emotional well-being?". The Humanistic Psychologist, 26, 243-274.

Ferrer-I-Carbonell, A. y Gowdy, J. M. (2007) "Environmental degradation and happiness". Ecological Economics, 60(3), 509-516.

FıøRTOFт, I. (2004) "Landscape as playscape: The effects of natural environments on children's play and motor development". Children Youth and Environments, $14(2), 21-44$.

GleEson, B. (2012) "Critical Commentary. The Urban Age Paradox and Prospect". Urban Studies, 49(5), 931-943.

HAINES-YOUNG, R., y POTSCHIN, M. (2010) "The links between biodiversity, ecosystem services and human well-being". Ecosystem Ecology: a new synthesis, 1, 110-139.

Hamilton, K. y Clemens, M. (1999) "Genuine savings rates in developing countries". The World Bank Economic Review, 13(2), 333-356.

HUNTINGTON, H. P. (2000) “Using traditional ecological knowledge in science: methods and applications". Ecological applications, 10(5), 1270-1274.

IPCC (Intergovernmental Panel on Climate Change) (2007) Climate change 2007: Synthesis report. IPCC: Geneva.

JaX, K. y HeINK, U. (2015) "Human Well-Being (draft)", en M. Potschin y K. JaX (Eds.), OpenNESS Ecosystem Service Reference Book. EC FP7 Grant Agreement $n^{\circ} 308428$. 
KAHN, P. H. y KelleRt, S. R. (2002) Children and nature: Psychological, sociocultural, and evolutionary investigations. MIT press.

KAPLAN, R. y KAPLAN, S. (1989) The experience of nature: A psychological perspective. CUP Archive.

KAPLAN, R. (1992) "The psychological benefits of nearby nature", en D. Relf (Ed.), The Role of horticulture in human well-being and social development: A National Symposium, pp. 125-133. Arlington: Timber Press.

KELERT, S. R. (2005) Building for life: Designing and understanding the human-nature connection. Island press.

Kellert, S. R., y WILSON, E. O. (Eds.) (1995) The biophilia hypothesis. Island Press.

KING, M. F., ReNó, V. F., y Novo, E. M. (2014) "The concept, dimensions and methods of assessment of human well-being within a socioecological context: a literature review". Social indicators research, 116(3), 681-698.

KJELL, O. N. (2011) "Sustainable well-being: A potential synergy between sustainability and well-being research". Review of General Psychology, 15(3), 255.

KorpelA, K. y KINNUNEN, U. (2010) "How is leisure time interacting with nature related to the need for recovery from work demands? Testing multiple mediators". Leisure sciences, 33(1), 1-14.

Krueger, A. B., Kahneman, D., Fischler, C., Schkade, D., Schwarz, N. y Stone, A. A. (2009) "Time use and subjective well-being in France and the US". Social Indicators Research, 93(1), 7-18.

Kuo, F. E. y Suluvan, W. C. (2001) "Environment and crime in the inner city does vegetation reduce crime?". Environment and behavior, 33(3), 343-367.

Kuo, F. E. (2011) "Parks and Other Green Environments: 'Essential Components of a Healthy Human Habitat'". Australasian Parks and Leisure, 14(1), 10.

LE BILLN, P. (2001) "The political ecology of war: natural resources and armed conflicts". Political geography, 20(5), 561-584.

Leather, P., Pyrgas, M., Beale, D. y Lawrence, C. (1998) "Windows in the workplace sunlight, view, and occupational stress". Environmentand Behavior, 30(6), 739-762. 
LEWIS, C. A. (1996) Green nature/human nature: the meaning of plants in our lives. University of Illinois Press.

Louv, R. (2008) Last child in the woods: Saving our children from nature-deficit disorder. Algonquin Books.

MA (Miliennium Ecosystem Assessment) (2003) Ecosystems and human well-being; a framework for assessment. Island Press.

- (2005a) Ecosystems and human well-being: Synthesis. Island Press.

- (2005b) Ecosystems and human well-being: Biodiversity synthesis. World Resources Institute.

Mats, J., Verheis, R. A., de Vries, S., Spreeuwenberg, P., Schellevis, F. G. y GroeneweGEN, P. P. (2009) "Morbidity is related to a green living environment". Journal of epidemiology and community health, 63(12), 967-973.

Maller, C., Townsend, M., Brown, P. y St Leger, L. (2002) Healthy parks, healthy people: the health benefits of contact with nature in a park context: a review of current literature. Deakin University Faculty of Health y Behavioural Sciences.

Markandya, A., Taylor, T., Longo, A., Murty, M. N., Murty, S. y Dhayala, K. (2008) "Counting the cost of vulture decline - An appraisal of the human health and other benefits of vultures in India". Ecological Economics, 67(2), 194-204.

MARTíN-López, B. y MONTES, C. (2014) "Restoring the human capacity for conserving biodiversity: a social-ecological approach". Sustainability Science, 1-8.

McCauley, D. J. (2006) "Selling out on nature". Nature, 443(7107), 27-28.

McMichael, A., Scholes, R., Hefny, M., Pereira, E., Palm, C. y Foale, S. (2005) "Linking ecosystem services and human well-being", en D. CAPISTRANo y C. SAMPER (Eds.), Sub-global assessments of the Millennium Ecosystem Assessment, pp. 43-60. Washington, DC: Island Press.

MONTES, C. y DuQUe, M. (2013) "Una aproximación al concepto de resiliencia urbana en el Antropoceno". Flora Capital, 13, 7-10.

MOONeY, H. A., CROPPER, A. y Reid, W. (2004) "The millennium ecosystem assessment: what is it all about?". Trends in ecology y evolution, 19(5), 221-224. 
NisBet, E. K., ZelensKI, J. M. y MuRPHY, S. A. (2008) "The nature relatedness scale: Linking individual s' connection with nature to environmental concern and behavior". Environment and Behavior, 41(5), 715-740

- (2011) "Happiness is in our nature: Exploring nature relatedness as a contributor to subjective well-being". Journal of Happiness Studies, 12(2), 303-322.

Pain, D. J., Cunningham, A. A., Donald, P. F., Duckworth, J. W., Houston, D. C., Katzner, T., Parry-Jones, J., Poole, C., Prakash, V., Round, P. y Timmins, R. (2003) "Causes and effects of temporospatial declines of Gyps vultures in Asia". Conservation Biology, 17(3), 661-671.

PolshCHUK, Y. y RausChmaYer, F. (2012) "Beyond "benefits"? Looking at ecosystem services through the capability approach". Ecological Economics, 81, 103-111.

Prakash, V., Pain, D. J., Cunningham, A. A., Donald, P. F., Prakash, N., Verma, A., Gargl, R., Sivakumar, S. y Rahmani, A. R. (2003) "Catastrophic collapse of Indian white-backed Gyps bengalensis and long-billed Gyps indicus vulture populations". Biological conservation, 109(3), 381-390.

Roberts, L.; Brower, A.; KerR, G.; Lambert, S.; McWilliam, W.; Moore, K.; Quinn, J.; Simmons, D.; Thrush, S.; TOWnsend, M.; Blaschke, P.; Costanza, R.; Cullen, R.; Hughey, K. y WRATEN, S. (2015) The nature of wellbeing: how nature's ecosystem services contribute to the wellbeing of New Zealand and New Zealanders. Department of Conservation, Wellington.

ROBERTSON, E. (2008) Medicinal plants at risk. Nature's pharmacy, our treasure chest: Why we must conserve our natural heritage. A Native Plant Conservation Campaign Report. Center for Biological Diversity, Tucson, US.

Rockström, J., Steffen, W. L., Noone, K., Persson, Å., Chapin III, F. S., Lambin, E., ... y NYKVIST, B. (2009) "Planetary boundaries: exploring the safe operating space for humanity". Ecology and Society, 14(2), 32.

ROHDE, C. L. E. y KENDLE, A. D. (1994) Human well-being, natural landscapes and wildlife in urban areas: a review. English Nature.

Salzman, J., Thompson JR., B. H. y Dally, G. C. (2001) "Protecting ecosystem services: Science, economics, and law". Stanford Environmental Law Journal, 20, 309. 
Sandifer, P. A., Sutton-Grier, A. E., y Ward, B. P. (2015) "Exploring connections among nature, biodiversity, ecosystem services, and human health and well-being: Opportunities to enhance health and biodiversity conservation". Ecosystem Services, 12, 1-15.

SIIVA, J. y BROWN, Z. (2013) "More than the sum of their parts: Valuing environmental quality by combining Life Satisfaction Surveys and GIS data". OECD Statistics Working Paper, 50.

Smith, L. M., Case, J. L., Smith, H. M., Harwell, L. C. y Summers, J. K. (2013) "Relating ecoystem services to domains of human well-being: Foundation for a US index". Ecological Indicators, 28, 79-90.

SONNENTAG, S., BINNEWIES, C. y MoJZA, E. J. (2010) "Staying well and engaged when demands are high: the role of psychological detachment". Journal of Applied Psychology, 95(5), 965.

Steffen, W., Sanderson, A., Tyson, P. D., Jäger, J., Matson, P. A., Moore III, B. Y OLDFIELD, F. (2004) Global change and the earth system: a planet under pressure. Nueva York.

Summers, J. K., SMith, L. M., CASE, J. L. y LinthuRSt, R. A. (2012) "A review of the elements of human well-being with an emphasis on the contribution of ecosystem services". Ambio, 41(4), 327-340.

Summers, J. K., Smith, L. M., Harwell, L. C., Case, J. L., Wade, C. M., Straub, K. R. Y SMITH, H. M. (2014) "An Index of Human Well-Being for the US: A TRIO Approach". Sustainability, 6(6), 3915-3935.

TAINTER, J. (1990) The collapse of complex societies. Cambridge University Press.

Takano, T., Nakamura, K. y Watanabe, M. (2002) “Urban residential environments and senior citizens' longevity in megacity areas: the importance of walkable green spaces". Journal of epidemiology and community health, 56(12), 913-918.

TAYLOR, A. y KUO, F. E. (2009) "Children with attention deficits concentrate better after walk in the park". Journal of Attention Disorders, 12, 402-409.

ULRICH, R. (1984) "View through a window may influence recovery". Science, 224(4647), 224-225. 
Van den Berg, A. E., Hartig, T. y StaAts, H. (2007) "Preference for nature in urbanized societies: Stress, restoration, and the pursuit of sustainability". Journal of social issues, 63(1), 79-96.

Van den Berg, A. E., MaAs, J., Verhelu, R. A. y Groenewegen, P. P. (2010) "Green space as a buffer between stressful life events and health". Social science $y$ medicine, 70(8), 1203-1210.

Vemuri, A. W. y Costanza, R. (2006) "The role of human, social, built, and natural capital in explaining life satisfaction at the country level: Toward a National WellBeing Index (NWI)". Ecological Economics, 58(1), 119-133.

Wels, N. M. (2000) "At home with nature effects of "greenness" on children's cognitive functioning". Environment and behavior, 32(6), 775-795.

Wells, N. M. y Evans, G. W. (2003) "Nearby nature a buffer of life stress among rural children". Environment and behavior, 35(3), 311-330.

WelsCH, H. (2006) "Environment and happiness: Valuation of air pollution using life satisfaction data". Ecological Economics, 58(4), 801-813.

White, M. P., Alcock, I., Wheeler, B. W. y Depledge, M. H. (2013) "Would you be happier living in a greener urban area? A fixed-effects analysis of panel data". Psychological science, 24, 920-928.

Willams, D. R. y Patterson, M. E. (2008) "Place, Leisure, and Well-being", en J. EYLES y A. WiLIAMS (Eds.) Sense of Place, Health and Quality of Life, pp. 105-119. Aldershot, Ashgate Publishing Ltd.

WıIsON, E. O. (1984) Biophilia. Harvard. Press, Cambridge (Mass.)

WINTER, D. y Koger, S. D. (2004) Psychology of everyday problems. 